\title{
Rasgos de personalidad y autoestima en víctimas de acoso laboral ${ }^{*}$
}

\section{Personality features and self-esteem in job relentless persecution victims}

\author{
John Alcides Camargo \\ Alexandra Puentes Suárez ${ }^{* *}$ \\ Universidad Pedagógica \\ y Tecnológica de Colombia \\ Tunja, Colombia \\ Recibido: 24 de julio de 2009 \\ Revisado: 29 de agosto de 2009 \\ Aceptado: 30 de octubre de 2009
}

\section{Resumen}

La investigación describe las conductas de acoso psicológico laboral, rasgos de personalidad y autoestima de personas que han denunciado acoso laboral ante instancias legales. Participaron cuatro mujeres, entre 45 y 57 años de edad, con tiempo de vinculación mayor a 15 años en organizaciones públicas. Se aplicó el cuestionario Lipt-60, la prueba BFQ, la escala de autoestima de Rosenberg y una entrevista semiestructurada.

Los resultados reflejan la presencia de acoso psicológico laboral vertical descendente, cada caso es diferente al no presentar semejanzas en las acciones hostigadoras percibidas y difieren en la intensidad y frecuencia de las estrategias utilizadas por el acosador hacia la víctima. En los rasgos de personalidad todos los casos presentan puntuaciones bajas en afabilidad y estabilidad emocional.

Palabras clave: acoso psicológico laboral, rasgos de personalidad, autoestima, víctimas de acoso laboral.

Artículo de investigación. Línea de investigación Caracterización, salud y trabajo en organizaciones formales e informales, del Grupo de Investigación Salud, Trabajo y Calidad de Vida, Facultad Ciencias de la Salud, Universidad Pedagógica y Tecnológica de Colombia.

Correspondencia: Alexandra Puentes Suárez. Universidad Pedagógica y Tecnológica de Colombia, Tunja. Correo electrónico: alexaps2@yahoo.es. Dirección postal: Unidad de Política Social, Avenida Central del Norte, UPTC, Tunja, Colombia. 


\section{Abstract}

The current research aims at describing behaviors dealing with the job psychological relentless persecution as well as features of personality and self-esteem in people who have been victims and have been able to accuse this complex issue in front of legal instances. The participants were four women aged between forty-five and fifty-seven years old, with time of entailment over fifteen years, at public organizations. The research instruments applied were the Lipt-60 questionnaire, the BFQ test, the Rosenberg self-esteem scale and a semi-structured interview, too. The outcomes clearly reflect the presence of descending-vertical job psychological relentless persecution; each case is different from the other since they do not show similarities in the harassing actions and are also different regarding the intensity and the frequency of the strategies used by the pursuer towards the victim. Finally, in regard to the personality features, all cases present low fluctuations of affability and emotional stability.

Key words: Job psychological relentless persecution, personality features, self-esteem, and job relentless persecution.

\section{Introducción}

Según la Organización Internacional del Trabajo (OIT, 2000), en el mundo los riesgos ocupacionales ocasionan 270 millones de accidentes y cada año hay dos millones de muertes. Al año se presentan 160 millones de enfermedades profesionales no mortales, de las cuales el $10 \%$ son enfermedades mentales ocasionadas por factores de riesgos ocupacionales de tipo psicosocial, entre los que se considera la violencia en el trabajo (acoso moral, hostigamiento, aislamiento, amedrentamiento $y$ acoso sexual).

En la tercera Encuesta Europea sobre condiciones de trabajo, publicada por la OIT (2000), un 9\% de los trabajadores europeos han sido víctimas de mobbing o acoso psicológico en el trabajo (13 millones de personas). En España el porcentaje se sitúa en torno al $5 \%$, lo que cuesta a la sociedad más de 90 millones de euros, por bajas o invalidez profesional.

De manera similar, Leymann (1996) citado por Hirigoyen (2001), establece que en Suecia el hostigamiento psicológico en el trabajo origina entre el $10 \%$ y el $15 \%$ de los suicidios registrados, sin tener en cuenta los casos que no son denunciados por temor a las diferentes represarias o repercusiones que puedan sufrir las víctimas.
Colombia no es ajena a esta realidad mundial, según el estudio realizado en el año 2004 por el Ministerio de Protección Social y la Universidad de Antioquia, en los sectores de vigilancia priva$\mathrm{da}$, transporte, salud y finanzas, se plantea que el acoso laboral es un fenómeno social que atenta contra la salud, seguridad de las personas y afecta la calidad de vida. De igual forma, se reconoce que para el "[...] 9\% de las personas encuestadas, esta experiencia se traduce, de alguna manera, en la aparición de consecuencias mentales que no le permiten alejarse totalmente de la experiencia de agresión" (p. 41).

Por otro lado, según Peralta (2006), en un estudio de caso sobre las manifestaciones del acoso laboral, encuentra que: "las víctimas de acoso laboral son personas que presentan síntomas asociados al estrés postraumático, que incapacitan y lesionan psicológicamente a las personas de manera grave" (p. 21).

Los daños psicológicos asociados a la presencia de acoso psicológico laboral, a largo plazo, pueden conducir a depresión, ansiedad, baja autoestima, pérdida de interés por el trabajo, fallos de la memoria, frustración, alteraciones emocionales, problemas físicos, conductuales, familiares, sociales y como etapa final en muchos casos, el 
suicidio (Martínez \& Cruz, 2008). Además, este problema es causa de un elevado costo en términos económicos y sociales (Lutgen-Sandvik, 2003).

Ante el problema de acoso psicológico laboral, se evidencia el desconocimiento de la norma, la escasa política de prevención e intervención ocupacional, médica y psicoterapéutica en las organizaciones y en el individuo, reconociendo que es un malestar general en el mundo laboral importante de analizar. Es así que, conocer las dimensiones que más inciden en el acoso psicológico, la autoestima que presenta la víctima, y los rasgos de personalidad que manifiestan los sujetos después del acoso psicológico laboral, permitirá entender el fenómeno desde la realidad percibida por los afectados.

\section{Acoso laboral}

El acoso psicológico laboral o mobbing es considerado como el abuso emocional asociado a conductas agresivas y hostiles que se ejercen de manera constante entre compañeros de trabajo o entre superiores y subordinados, acompañadas de comunicaciones negativas y poco éticas. El acoso laboral se dirige de manera sistemática desde uno o varios individuos hacia un solo individuo, quien debido a las continuas actividades de hostigamiento se ve obligado a defenderse de manera infructuosa de aquellos que lo atacan (Leymann, 1996; Einarsen, 2000; Hirigoyen, 2001; Piñuel \& Zabala, 2001; Boada, De Diego \& Vigil, 2003; citados por Peralta, 2006). Por tanto, se trata de un fenómeno que conjuga diferentes factores psicológicos, sociales, éticos y jurídicos con repercusiones culturales, económicas y políticas.

Por otra parte, la finalidad que tiene el acoso psicológico laboral es intimidar, apocar, reducir, aplanar, amedrentar y consumir emocional e intelectualmente a la víctima, con la intención de eliminarla de la organización o de satisfacer la necesidad insaciable del acosador: controlar, agredir y destruir (Piñuel \& Zabala, 2001).

Asimismo, se busca demostrar el poder que ostenta el acosador como medio para amedrantar al resto del personal, con el fin de mantenerse y afianzarse. Se trata de hacer cundir el psicoterror laboral entre los miembros de la organización, que tendrán que advertir lo que les podría ocurrir si no se someten a los dictados del grupo que los hostiga (Luna, 2003). Además, las ingeniosas o sutiles técnicas utilizadas persiguen agobiar, calumniar y atacar el trabajo, las convicciones y la vida privada de la víctima, aislándola, estigmatizándola y amenazándola (Trujillo, Valderrabano \& Hernández, 2007), todo como resultado de diferentes causas generadoras de comportamientos abusivos y hostigadores, así como de un clima deficiente en la organización.

Teniendo en cuenta las observaciones que realizan Piñuel \& Zavala (2003), la causa más frecuente del acoso psicológico laboral son los celos y la envidia que sienten los acosadores; la víctima suele ser envidiada por poseer alguna cualidad o rasgo que los hace destacar del resto de las personas que trabajan en la organización. El acoso psicológico laboral se puede desencadenar cuando la víctima no hace parte del grupo que maneja el statu quo de la organización y no permite ser manipulado por otro u otros miembros del grupo.

Leymann (1997) plantea que las estrategias que se utilizan para destruir a la víctima son: limitación a la comunicación, al contacto social, desprestigio ante los compañeros, descrédito de la capacidad laboral y profesional, y el compromiso de la salud; dimensiones que hacen parte del cuestionario Leymann Inventory of Psychological Terrorization (LIPT, denominado en la versión española LIPT-60 [González de Rivera, 2003]).

Al limitar la comunicación, a la víctima se le niega el derecho a expresarse o hacerse oír, además, el acosador impone con su autoridad aquello que se puede decir, bloqueando la libre expresión y el flujo continuo de información (González de Rivera, 2003). Los acosadores restringen los canales de comunicación de sus víctimas, comenzando por evitar comunicarse directamente con ellas y en la mayoría de los casos se le prohíbe dirigirse a sus compañeros, para ello se le puede separar del grupo al que pertenece asignándole una nueva ubicación (Luna, 2003). 
En la limitación del contacto social no se dirige la palabra a la víctima, procurando además que nadie lo haga, se le cortan las fuentes de información, se le aísla físicamente de sus compañeros y se utilizan conductas humillantes, limitándole las posibilidades de mantener contacto social. De igual manera, se causa desprestigio ante los compañeros, haciendo bromas y burlas sobre la víctima, su familia, orígenes, antecedentes y entorno (González de Rivera, 2003).

Por otro lado, se desacredita la capacidad laboral y profesional, al asignar a la víctima tareas muy por debajo o muy por encima de su capacidad, o no se le permite hacer nada; se le critican los más mínimos errores o defectos; se desprecia su trabajo y sus capacidades profesionales. En cualquier caso, sus trabajos van a ser siempre evaluados de forma negativa, creando en el trabajador una sensación de inutilidad, de no contar con las capacidades y habilidades necesarias para llevar a cabo las misiones encomendadas (Luna, 2003).

Todo lo anterior lleva a serios compromisos en la salud de quien es víctima de acoso laboral, además de los ataques directos, el sometimiento a un régimen de acoso psicológico ya tiene efectos negativos, psicológicos y psicosomáticos (González de Rivera, 2003). Algunos autores han mostrado que el Mobbing reduce la salud física y psicológica de sus víctimas y afecta negativamente al bienestar y la eficiencia de otros empleados (Matthiensen \& Einarsen, 2001, Piñuel, 2001). La víctima puede llegar a presentar efectos a nivel cognitivo, como pérdida de memoria, inseguridad, dificultades para la concentración; efectos psicosomáticos, como dolor de cabeza y espalda; efectos físicos, dolores cervicales, fibromialgia; y trastornos del sueño, entre otros.

Además de las consecuencias señaladas en el área de la salud, autores como Piñuel (2001) destacan que el acoso laboral puede ocasionar un gran deterioro en la víctima a nivel social, familiar e interpersonal. Asimismo, se destruye la identidad, provocando cambios de personalidad, tornándose confusa, menos eficiente y con un alto nivel de miedo, culpa y vergüenza, de este modo afecta no sólo el trabajo sino su comportamiento, salud mental y las relaciones interpersonales (Piñuel \& Zavala, 2001).

Los cambios que va sufriendo la víctima de acoso laboral, en su personalidad, acontece a una serie de factores psicológicos y a repercusiones físicas individuales que la persona toma como intentos vanos de solución en el medio laboral donde es hostigado, complicando su estado de ánimo, cognitivo, físico y contextual; lo que repercute en el deterioro de la salud mental y física, síntomas que son evidentes en los cambios bruscos de personalidad que va presentando el individuo (Luna, 2003).

En el contexto de la personalidad, la teoría de los rasgos sostiene que los patrones característicos del comportamiento, pensamiento y sentimiento son los resultados de los rasgos del individuo y hacen énfasis en las diferencias individuales de las características que son más o menos estables a través del tiempo y de las situaciones (Cloninger, 2003).

Así, las teorías de la personalidad se dedican a estudiar los factores determinantes de la misma y los agentes que llevan a la persona a actuar como lo hace, además, establece indicadores para poder predecir conductas (Feldman, 2002).

Dentro de esta línea se adopta el modelo de los cinco factores, también denominado los "cinco grandes", el cual es un sistema que identifica las cinco dimensiones de la personalidad, como un intento de unificación entre los distintos puntos de vista existentes hasta el momento, proponiendo cinco dimensiones fundamentales para su descripción y evaluación: extraversión/energía, amabilidad/afabilidad, tesón/rectitud, inestabilidad emocional o neuroticismo e intelecto/apertura a la experiencia (Costa \& McCrae, 2001).

Para Coon (2005) cualquier rasgo que se imagine, se relacionará con uno de los cinco factores, permitiendo predecir cómo se comportarán las personas en diversas circunstancias, con el objetivo principal de describir la personalidad y no sus causas (Cloninger, 2003). 
Teniendo en cuenta los rasgos de personalidad, la víctima puede manifestar diferentes maneras de responder ante el acoso psicológico laboral: reacciones emocionales de miedo, las cuales puede conducirlo a un juego de roles y asumir conductas diferentes a su propio estilo, como actuar agresivamente; también se presenta enojo, paranoia, ansiedad, depresión, dolor, desesperación o vulnerabilidad (Ireland \& Snowden, 2002). Rasgos que se pueden evidenciar y acentuar, cuando la persona carece de intercambio social en su vida diaria, concibiéndose como una persona insegura, al ser señalada por los demás y por la forma en que los otros lo identifican y lo perciben (Goffmann, 1996).

Sin embargo, Coon (2005) considera que los rasgos interactúan con la situación para determinar la conducta futura, y las interacciones entre ambos se dan cuando las circunstancias externas influyen en la expresión de los rasgos.

Por otra parte, la víctima de acoso laboral se va deteriorando profesional y psíquicamente hasta perder su capacidad de autoestima, luego cae en la depresión, enfermedades psicosomáticas, insomnio, alcoholismo e incluso, el suicidio (Góngora, Martín \& Rivas, 2002).

La autoestima es la evaluación global de una persona con respecto a su autovaloración o auto-imagen, siendo el componente afectivo del sí mismo, al representar los sentimientos del individuo y su evaluación con una ponderación de su propio valor en un continuo que va de lo positivo a lo negativo (Sánchez, 1999).

De igual forma, en el ámbito profesional, la autoestima positiva facilita una mejor percepción de la realidad y optimiza la comunicación interpersonal, ayuda a tolerar mejor el estrés, la incertidumbre y a vivir los procesos de cambio (Pando, Aranda, Aldrete \& Reynaga, 2006). Siendo un factor necesario para el afrontamiento que pueda realizar la víctima del acoso psicológico laboral.

Así, se hace necesario estudiar el fenómeno desde la perspectiva de las víctimas que han denunciado su caso ante instancias legales, identificando las conductas de acoso psicológico laboral, los rasgos de personalidad y nivel de autoestima; buscando entender la gran complejidad de este fenómeno.

\section{Método}

\section{Diseño}

La investigación corresponde a un estudio de caso, con el cual se busca medir y describir las conductas de las personas involucradas en el fenómeno del acoso laboral (Martínez, 2006; Hernández, Fernández \& Baptista, 2006).

\section{Participantes}

En el estudio participaron cuatro mujeres, con edades comprendidas entre los 45 y 57 años, empleadas del sector público, con contratos a término indefinido. La selección se hizo por conveniencia, teniendo en cuenta los siguientes criterios de inclusión: personas víctimas de acoso laboral por un período mínimo de seis meses, que hayan denunciado su caso ante una instancia legal. Se da la participación de manera voluntaria y firma del respectivo consentimiento informado, teniendo en cuenta los criterios de investigación científica planteados en la Ley 1090 de 2006. 
Tabla 1. Características demográficas de los participantes

\begin{tabular}{|c|c|c|c|c|}
\hline Características demográficas & Caso 1 & Caso 2 & Caso 3 & Caso 4 \\
\hline Edad & 53 & 53 & 57 & 45 \\
\hline Estado civil & Separada & Casada & Casada & Separada \\
\hline Número de hijos & 3 & 4 & 1 & 2 \\
\hline Escolaridad & Técnico & Bachiller & Posgrado & Posgrado \\
\hline Ocupación & Secretaria & Secretaria & Docente & Docente \\
\hline Antigüedad en el cargo & 26 años & 31 años & 31 años & 15 años \\
\hline Organización donde labora & Juzgado & Juzgado & Colegio & Colegio \\
\hline Acosador & $\begin{array}{l}\text { Jefe inmediato } \\
\text { (Juez) }\end{array}$ & $\begin{array}{l}\text { Jefe inmedia- } \\
\text { to (Juez) }\end{array}$ & $\begin{array}{l}\text { Jefe Inmediato } \\
\text { (Rector) compa- } \\
\text { ñeros }\end{array}$ & $\begin{array}{l}\text { Jefe inmediato } \\
\text { (Rector) }\end{array}$ \\
\hline Tipo de acoso & Vertical & Vertical & $\begin{array}{l}\text { Vertical } \\
\text { Horizontal }\end{array}$ & Vertical \\
\hline Diagnóstico & Depresión & No reporta & Depresión & Depresión \\
\hline Tiempo de acoso & Un año & Un año & Nueve años & Un año \\
\hline Instancia ante la cual denuncia & $\begin{array}{l}\text { Consejo Supe- } \\
\text { rior de la Judi- } \\
\text { catura }\end{array}$ & Juzgado & $\begin{array}{l}\text { Coordinador } \\
\text { Rector } \\
\text { Director de nú- } \\
\text { cleo } \\
\text { Gobernación } \\
\text { Secretaría De- } \\
\text { partamental de } \\
\text { Educación }\end{array}$ & $\begin{array}{l}\text { Secretaría De- } \\
\text { partamental de } \\
\text { Educación }\end{array}$ \\
\hline
\end{tabular}

\section{Instrumentos}

Cuestionario de estrategias de acoso en el trabajo LIPT-60 (Leymann Inventory of Psychological Terrorization Modificado, González de Rivera \& Rodríguez, 2003). Proporciona una información global como la frecuencia e intensidad percibida del conjunto de las estrategias de acoso padecidas, y una particular para el análisis de las respuestas. Consta de seis subescalas de acoso: Desprestigio laboral, Entorpecimiento del progreso, Bloqueo de la comunicación, Intimidación encubierta, Intimidación manifiesta y Desprestigio personal.

Desprestigio laboral (DL). Constituye aquellas estrategias de acoso en el trabajo, en las que se produce un descrédito o desprestigio laboral, bien, a través de distorsión en la comunicación, como rumores o calumnias, o con medidas restrictivas o de agravio comparativo con el resto de los trabajadores, minimizando u ocultando sus logros.

Entorpecimiento del progreso (EP). Bloqueo sistemático de la actividad laboral, degradando al trabajador con tareas inapropiadas en la forma o en el contenido, de acuerdo con sus competencias.

Bloqueo de la comunicación (BC). Bloqueo de la comunicación intraorganizacional (dentro de la organización) y extraorganizacional (con el exterior de la organización).

Intimidación encubierta (IE). Amenazas y daños encubiertos, que predominantemente no dejan 
"huella" o se realizan de manera "limpia", sin que se puedan delimitar responsables específicos.

Intimidación manifiesta (IM). Amenazas o restricciones que se imponen a la víctima de forma directa, no disimulada, incluso en público, tales como: amenazas verbales, gritos o hacerla quedar en ridículo.

Desprestigio personal (DP). Descrédito o desprestigio de su vida personal y privada (no laboral), mediante la crítica, burla y agravio de su forma de ser, vivir y pensar.

Este cuestionario es una adaptación del realizado por Leyman (1984), actualmente se ha adaptado a la versión española con 60 ítems. Para el cálculo de los índices de fiabilidad se ha utilizado el método de correlación de las dos mitades y el alfa de Cronbach, teniéndose una fiabilidad de 0,83 en cada una de las subescalas.

Prueba BIG FIVE o Cinco factores de personalidad (BFQ). Cuestionario con 132 elementos de respuesta múltiple (tipo Likert) para identificar las cinco dimensiones fundamentales de la personalidad humana: Energía (E), Afabilidad (A), Tesón $(T)$, Estabilidad emocional (EE), Apertura mental (AM), y la escala de Distorsión (D).

Energía $(E)$. Inherente a una visión confiada y entusiasta de múltiples aspectos de la vida, principalmente de tipo interpersonal. Permite predecir varias conductas sociables de la personalidad.

Afabilidad (A). Preocupación de tipo altruista y de apoyo emocional a los demás. Manifiesta una personalidad amistosa y complaciente, que evita la hostilidad y tiende a llevarse bien con los demás.

Tesón $(\mathrm{T})$. Propia de un comportamiento de tipo perseverante, escrupuloso y responsable. También nombrada seriedad, control de impulsos y voluntad del logro, al describir diferencias en el orden y autodisciplina de la gente.

Estabilidad emocional (EE). Rasgo de amplio espectro, con características tales como capacidad para afrontar los efectos negativos de la ansie- dad, depresión, irritabilidad o frustración. Describe el nivel neurótico en el que se encuentra una persona, así como el control emocional y fortaleza del yo.

Apertura mental (AM). Reconociendo a este factor con los términos artístico, curioso, imaginativo, intuitivo, original y de amplias facultades, sobre todo de tipo intelectual ante nuevas ideas, valores, sentimientos e intereses.

La escala de Distorsión (D) consta de 12 elementos y tiene por finalidad proporcionar una medida de la tendencia a ofrecer un perfil falseado de sí mismo al responder al cuestionario (tipos de perfil que evalúa: realista, investigador, creativo, social, dirigente y convencional).

En cada uno de los cinco factores se han identificado dos subdimensiones, cada una de las cuales hace referencia a distintos aspectos de la propia dimensión. En cada subdimensión (integrada por 12 elementos) la mitad de las afirmaciones han sido formuladas en sentido positivo con respecto al nombre de la escala, mientras la otra mitad está formulada en sentido negativo, con el fin de controlar eventuales fenómenos de sesgos de respuesta.

El Apha en las dimensiones es de 0.72 para Energía, Afabilidad $(0,75)$, Tcon $(0,76)$, estabilidad emocional (0.66) y Apertura mental (0.64).

Escala de Autoestima de Rosenberg (RSES). Consta de 10 preguntas que se refiere a la valoración y aceptación que el individuo hace y que acostumbra a mantener con respecto a sí mismo, la cual se expresa en una actitud de aprobación o desaprobación (Rosenberg, 1965; citado por Vargas \& Barera, 2005). Los autores del cuestionario no han establecido puntos de corte, es decir, puntuaciones que permitan clasificar el tipo de autoestima según la puntuación obtenida, aunque se suele considerar como rango normal de puntuación el situado entre 25 y 35 puntos (Martín-Albo, Núñez, Navarro \& Grijalvo, 2007).

Sin embargo, se toma una puntuación mínima de 10 y máxima de 40 , diferenciando entre una autoestima elevada considerada como normal (30- 
40), media (26-29) y baja (10-20), la escala presenta un coeficiente alfa de Cronbach de 0.86 , y su fiabilidad test-retest de 0.85 (Cortés \& Justicia, 2006).

\section{Procedimiento}

A través de diferentes fuentes de información, se logra el acercamiento con las personas que han sido víctimas de acoso laboral y han denunciado su caso a una instancia legal. Luego se habla con cada uno de los participantes, se les informa sobre el objetivo del estudio, y se procede a la firma del consentimiento informado por cada una de las personas. Los investigadores diligenciaron la ficha demográfica y aplicaron el cuestionario LIPT-60, la prueba Big Five (BFQ) y la escala RSES de autoestima. Luego se procedió a realizar la calificación de cada una de las pruebas y el análisis de los resultados, tomando cada uno de los casos de manera independiente y estableciendo un análisis general del fenómeno.

\section{Resultados}

Se presentan los resultados obtenidos, en cada una de las variables de estudio: acoso psicológico laboral, rasgos de personalidad y autoestima; se destacan los datos más relevantes para cada uno de lo cuatro casos valorados.

\section{Índices globales y dimensionales de acoso psicológico laboral}

En la Figura 1 se muestran las puntuaciones obtenidas en cada una de las estrategias de acoso reportadas por las víctimas, identificas así: NEAP: Número total de estrategias de acoso psicológico tolerado, IGAP: Índice global de acoso psicológiCo, IMAP: Índice medio de intensidad de acoso psicológico, DL: Desprestigio laboral, EP: Entorpecimiento del progreso, BC: Bloqueo de la comunicación, IE: Intimidación encubierta, IM: Intimidación manifiesta y, DP: Desprestigio personal.

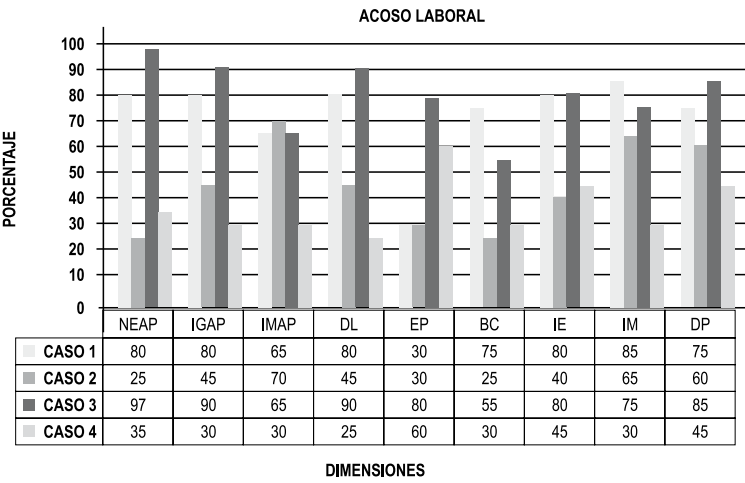

Figura 1. Índices globales y dimensiones afectadas por acoso psicológico laboral.

A nivel global, los casos con mayor número de estrategias de acoso psicológico percibidos, corresponden al individuo del caso 3 con un $97 \%$ y el caso 1 con un $80 \%$ de conductas abusivas y hostiles percibidas en el medio laboral, indicando una alta amplitud de ocurrencia al exhibir diferentes comportamientos de acoso. El caso 4 presenta una moderada amplitud de ocurrencia con un 35\% de comportamientos de acoso y en menor porcentaje el caso 2 con $25 \%$ de estrategias de acoso percibidas por la víctima.

Frente al índice global de acoso psicológico los individuos que perciben mayor afectación, son el caso 3 en un $90 \%$ y el caso 1 un $80 \%$, de acciones que combinan las diferentes conductas de acoso y la intensidad de las mismas, evidenciando un nivel elevado de estrategias de maltrato laboral experimentadas por la víctima.

Los casos que presentan una elevada intensidad en las diferentes conductas de acoso percibidas por la víctima, corresponden al caso 2 con un $70 \%$, en el cual el individuo percibió pocas conductas de acoso, pero con una elevada intensidad; al igual que el caso 1 y el caso 3 que reportaron un $65 \%$, en esta escala.

Es así como la forma de acoso psicológico más experimentada en el caso 1 corresponde a la intimidación manifiesta con un $85 \%$ de conductas hostiles, abusivas y amenazantes, realizadas en voz alta y delante de los compañeros; estas acciones están asociadas a conductas encubiertas 
y acciones de acoso psicológico que buscan desprestigiar laboralmente a la víctima.

Para el caso 2, las estrategias de acoso percibido con mayor intensidad y frecuencia son, las intimidaciones manifiestas con un $65 \%$; sin embargo, es frecuente encontrar conductas dirigidas a desprestigiar personalmente a la víctima. En el caso 3, se evidencia una mayor incidencia en las conductas dirigidas a atentar contra el prestigio laboral de la víctima, manifestando un $90 \%$ de ocurrencia; igualmente, se presenta una alta incidencia en las dimensiones de desprestigio personal, entorpecimiento del progreso, uso de intimidaciones encubiertas y manifiestas, afectando intensamente la salud mental y física de la víctima, así como a la organización misma.

En el caso 4, las conductas de acoso que afectaron y se percibieron con mayor frecuencia hacen referencia a la dimensión de entorpecimiento del progreso con un $60 \%$, con acciones como el bloqueo sistemático de la actividad laboral, llegando a degradar al trabajador.

\section{Rasgos de personalidad}

Las figuras 2 y 3 , muestran los datos de las dimensiones y subdimensiones de la personalidad, en todos los casos se observa que ninguna de las dimensiones de los rasgos de personalidad presenta puntajes por encima del promedio, además se encuentran rasgos que a nivel global son muy bajos.

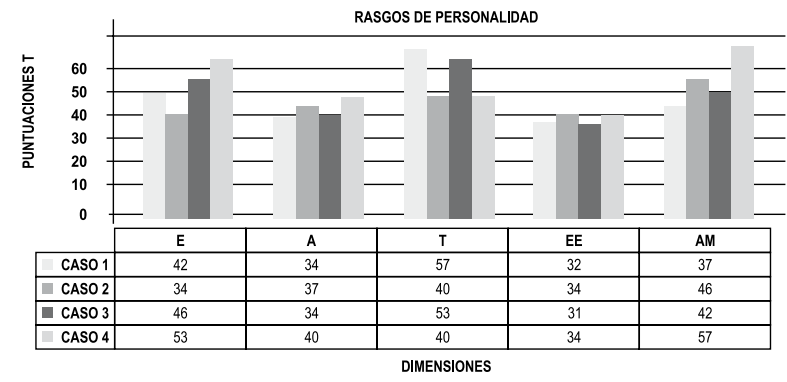

Figura 2. Dimensiones de la personalidad.

Nota: Energía (E), Afabilidad (A), Tesón (T), Estabilidad emocional (EE), y Apertura mental (AM).

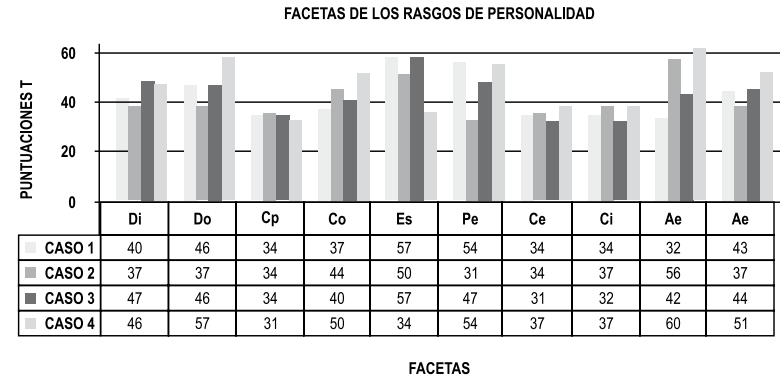

Figura 3. Subdimensiones de la personalidad. Nota: Di: Dinamismo, Do: Dominancia, Cp: Cooperación, Co: Cordialidad, Es: Escrupulosidad, Pe: Perseverancia, Ce: Control de emociones, Ci: Control de impulsos, Ac: Apertura a la cultura, Ae: Apertura a la experiencia.

Los cuatro casos tomados en el estudio presentan un nivel global bajo o muy bajo en la dimensión de Afabilidad, al igual que en sus subdimensiones de Cooperación y Cordialidad, puntuaciones que al parecer pueden reflejar que son personas irritables, suspicaces, poco cooperativas e insensibles. Probablemente presentan baja complacencia con los estímulos experimentados en su medio, tendiendo a desconfiar y desvalorar a los demás, con una alta probabilidad de presentar conflictos interpersonales, en los que pueden hacer uso del poder como una forma de solución de los problemas.

En la dimensión de Estabilidad Emocional, se observa que los cuatro casos presentan un nivel global muy bajo al igual que en los rasgos de Control de emociones y Control de impulsos. Evidenciando que probablemente pueden ser personas inestables emocionalmente con estados de ansiedad, tensión, generalmente aprensivos y con tendencia a la preocupación. Frecuentemente presentan labilidad emocional, con usuales períodos de insatisfacción e infelicidad con su vida y constante preocupación. 


\section{Autoestima}

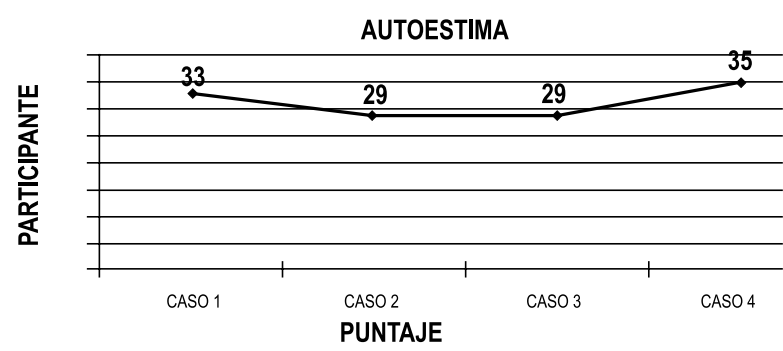

Figura 4. Puntuaciones obtenidas en la escala de autoestima.

Teniendo en cuenta los puntos de corte de la escala de autoestima de Rosenberg, se encuentra que el individuo del caso 1 presenta (33) puntos, el caso 2 (35) puntos, el caso 3 (29) puntos y el caso 4 (35) puntos; para todos los casos las personas manifiestan una valoración positiva acompañada de respeto por sí mismo y gran valía personal, percibida a partir de los pensamientos, sentimientos y sensaciones que experimentan sobre su autoimagen, que al no constituir valores extremos muestra una imagen de sí mismo y un autoconcepto acorde con la realidad sin propensiones al enaltecimiento, manteniendo el reconocimiento social o colectivo.

\section{Discusión}

El objetivo de esta investigación consistió en explorar y describir las conductas de acoso psicológico laboral, los rasgos de personalidad y el nivel de autoestima, en cuatro personas que fueron víctimas de acoso y denunciaron su caso ante instancias legales.

Es necesario resaltar que los datos encontrados en el estudio no se pueden generalizar a todas las víctimas de este fenómeno y son exclusivas de los casos estudiados. Según lo planteado por Piñuel (2001), muchos de los diagnósticos de las personas que consultan por acoso, son incorrectos en la medida que olvidan los aspectos situacionales que los están causando y que tienen su origen en una agresión externa, continuada, y mantenida, y no en la fragilidad constitucional de la víctima.
Así entonces, el caso 1 experimentó una elevada intensidad y frecuencia de conductas de acoso laboral, que en su mayoría son estrategias que buscaban intimidar en público, junto a comportamientos que desprestigiaron el área laboral y personal de la víctima, además de incomunicarla con el grupo de trabajo. Siendo algunas de las múltiples estrategias que puede emplear el acosador, quien hizo uso de comportamientos abusivos, como la exclusión de reuniones o de avance profesional, la humillación o el ridículo, la crítica constante en público, el castigo a otras personas por ser demasiado competentes y la generación de exceso de trabajo de forma injustificada con fines punitivos (Royal College of Nursing, 2005).

Los rasgos de personalidad que manifestó el caso 1 , y que destacan su comportamiento fueron las dimensiones de afabilidad y estabilidad emocional; evidenciando una persona poco cooperativa y cordial con el grupo de trabajo, además con la dificultad de controlar las emociones y los impulsos; cambios que son sustentados por Luna (2003), al reconocer que se debe a una serie de factores psicológicos y a repercusiones físicas e individuales, complicando el estado de ánimo, cognitivo, físico y contextual del individuo; síntomas que son evidentes en los cambios bruscos de personalidad que va presentando el acosado.

En el caso 2 se obtuvieron datos que evidencian sentimientos de miedo, apatía, repudio, arrogancia, acciones que interrumpen la comunicación intra-organizacional y un clima laboral deficiente, junto a una des-valoración del trabajo, que acompañado de otras conductas permitieron el desarrollo del acoso psicológico en la organización; el cual estuvo dirigido, especialmente, a generar desprestigio laboral y al uso de intimidaciones. Según Trujillo, Valderrabano \& Hernández (2007), las ingeniosas o sutiles técnicas utilizadas por el acosador, persiguen agobiar, calumniar y atacar el trabajo, las convicciones y la vida privada de la víctima, aislándola, estigmatizándola y amenazándola.

El caso 2 percibió pocas conductas de acoso psicológico laboral, con una alta intensidad en su exposición, presentando intimidaciones manifiestas acompañadas de gritos, críticas al desem- 
peño laboral y amenazas verbales; acciones que ocasionaron desprestigio personal del individuo por medio de rumores, y comentarios injustos, evaluando de forma negativa el trabajo, creando en la víctima una sensación de inutilidad, de no contar con las capacidades y habilidades necesarias para llevar a cabo las misiones encomendadas (Luna, 2003). La víctima adoptó una actitud pasiva, sumisa y tolerante frente a las agresiones psicológicas del acosador.

Se encontró en el caso 2, que los rasgos de personalidad destacados con niveles muy bajos, son energía, afabilidad y estabilidad emocional; evidenciándose como una persona ansiosa que manifiesta bajo control de impulsos y de emociones al ser vulnerable a las situaciones estresantes; perdiendo con facilidad la paciencia. Para Ireland \& Snowden (2002) las víctimas de acoso laboral pueden responder de diferentes maneras, como: reacciones emocionales de miedo, enojo, paranoia, desesperación o vulnerabilidad; en las cuales el miedo puede conducir al individuo a asumir conductas diferentes al propio estilo.

En el caso 3, se encontraron acciones que propician el acoso laboral en el medio educativo, con una trascendencia temporal de nueve años aproximadamente, en las que se ha presentado persecución, inequidad, hostigamiento, desprestigio e intimidaciones, cohibiendo el desenvolvimiento en el trabajo; asimismo, la discriminación, la inaceptación al diálogo, la acusación falsa, la desigualdad laboral, las amenazas, la violación de los derechos, entre otras; son acciones que se desarrollaron dentro de un contexto académico con la presencia de abuso de la autoridad y un clima laboral conflictivo.

De esta manera, el caso 3 presentó una elevada frecuencia e intensidad de conductas que ejercieron violencia psicológica extrema; se encontró que las acciones desprestigiaron laboral y personalmente a la víctima, entorpeciendo el progreso. Igualmente, la persona mostró una elevada afectación frente a las persistentes conductas de acoso psicológico percibidas, las cuales han sido intensas, degradando considerablemente la salud física y mental (IMAP de 65\% en el Lipt-60). Así, el acosador logró desgastar psicológicamente a la víctima hasta conseguir que se autoexcluyera de la organización (Góngora, Martín \& Rivas, 2002).

Dentro de los rasgos de personalidad, se percibió como una persona poco cooperativa y cordial con el grupo de trabajo, con dificultad para controlar impulsos y emociones frente a las relaciones interpersonales. Por tanto, el individuo que es aislado de los demás, carece de la retroalimentación del intercambio social en su vida diaria, se vuelve desconfiado, depresivo, hostil, ansioso y prevenido, concibiéndose como una persona insegura al ser señalada por los demás y por la forma en que los otros lo identifican y lo reciben (Goffmann, 1996).

Siguiendo la teoría de los rasgos, la cual habla de características individuales que son más o menos estables a través del tiempo y de las situaciones (Cloninger, 2003), se observa que la personalidad del individuo frente a las conductas de acoso laboral percibidas pueden variar como forma para afrontar las conductas de acoso (Luna, 2003).

En el caso 4 la causa principal del maltrato se debe a los celos profesionales y al sentimiento de inferioridad del acosador frente a la capacidad profesional del hostigado (Piñuel \& Zavala, 2003).

De esta forma, los efectos negativos que tiene el acoso en el ambiente de trabajo, ocasionan un deficiente clima laboral, interferencia en los canales de comunicación, descenso de la creatividad e innovación, despreocupación por la satisfacción de los clientes, la productividad, la atención al cliente, la satisfacción laboral; al igual que mayor absentismo, aumento de problemas en la salud de los trabajadores y el incremento de accidentes por descuido y desatención (Luna, 2003).

Igualmente, el empleo de acciones que buscan intimidar sin dejar huella de la conducta hostil realizada, buscando culpabilizar y demonizar al trabajador, quien en un momento dado cargará con la culpa de todo lo que sale mal (Trujillo, Valderrabano \& Hernández, 2007). Además, las implicaciones del acoso generaron consecuencias en la salud mental y física del participante, reduciendo la capacidad laboral, social e interacción familiar. 
Por otra parte, los rasgos de personalidad que manifiesta el individuo del caso 4 reflejan a una persona que se caracteriza por ser poco cooperativa, con baja escrupulosidad, siendo desorganizada en sus ideas y en las actividades que realiza, además, se le dificulta controlar sus emociones e impulsos, con tendencia a la preocupación, ansiedad y al enojo.

Asimismo, el acosador fue considerado como una persona dominante, que hace uso de su autoridad y abusa del poder conferido en el medio laboral, es paranoico, manifiesta desagrado por la víctima; de acuerdo con Piñuel \& Zavala (2003) la causa más frecuente del acoso psicológico laboral son los celos y la envidia que siente el victimario, buscando acabar con la persona acosada, haciéndola retirar de la organización o lograr dominarla según sus pretensiones.

Con relación a la autoestima, todos los participantes, manifiestan una valoración positiva, de respeto por sí mismo y de gran valía personal. Según Martínez \& Cruz (2008) los daños psicológicos asociados a la presencia de acoso psicológico laboral pueden conducir a una baja autoestima y al desarrollo de otros síntomas psicológicos, familiares y sociales. Sin embargo, puede considerarse que la intervención médica y psicoterapéutica, pudieron contribuir a una autovaloración positiva, permitiendo que en el ámbito profesional la autoestima facilite una mejor percepción de la realidad, una habilitada comunicación interpersonal, y mejores estrategias de afrontamiento ante el estrés (Pando, Aranda, Aldrete $\&$ Reynaga, 2006).

Esta investigación pretendió profundizar en el análisis del fenómeno del acoso psicológico laboral, desde la perspectiva de las víctimas; sin embargo, cabe resaltar que se hace necesario el análisis de un mayor número de casos, teniendo en cuenta las repercusiones en el área socio familiar, al igual que las estrategias de afrontamiento que utiliza la víctima, y las conductas individuales y grupales que mantienen este fenómeno.

\section{Referencias}

Boada, J., De Diego, R. \& Vigil, A. (2003). Mobbing: Análisis de las propiedades psicométricas y estructura factorial de cuatro escalas (MOBB-90; MOBBCF-21; MOBBCG-15 y MOBBCS-28). Revista Encuentros en Psicología Social, $1,5$.

Cloninger, S. (2003). Cattell y los cinco grandes. Teorías analítico-factoriales de los rasgos. Teorías de la personalidad, 229-269. México: Pearson Educación.

Coon, D. (2005). Personalidad. Fundamentos de Psicología. México: Thomson. Recuperado el 20/01/2009. En http://books.google.com.co/ books?id=xw

Cortés, D. \& Justicia, F. (2006). Afrontamiento del abuso sexual infantil y ajuste psicológico a largo plazo. Revista Psicothema, 20 (4), 509515. España.

Costa, P. T. \& McCrae, R. R. (2001). Inventario de Personalidad NEO revisado. Manual de aplicación. Madrid: TEA.

Einarsen, S. (2000). Harassment and bullying al work: A review of the Scandinavian approach. Aggression and violent behavior: Review Journal, 5 (4), 371-401.

Feldman, R. (2002). Personalidad. Psicología con aplicaciones en países con habla hispana. México: McGraw-Hill Interamericana.

Goffmann, G. (1996). Estigma: la identidad deteriorada. México: Paidós.

Góngora, J., Martín, M. \& Rivas M. (2002). Acoso Psicológico en el Trabajo "Mobbing”. España: Fondo de Publicaciones del Gobierno de Navarra.

González de Rivera, J.L. \& Rodríguez-Abuín, M. (2003). Cuestionario de estrategias de acoso psicológico: el LIP-60 (Leymann inventory of psychological terrorization) en versión española. Psiqui, 24 (2), 59-69. 
González de Rivera, L. (2003). Cuestionario de estrategias de acoso psicológico: El LIPT-60. Revista Psiquiatría, 32, 18-28.

Hernández, R., Fernández, C. \& Baptista, P. (2006). Metodología de la investigación (4 $4^{\mathrm{a}}$ ed.). Mexico: McGraw-Hill.

Hirigoyen, M. (2001). El acoso moral en el trabajo. Distinguir lo verdadero de lo falso. (p. 769). Madrid: Paidós.

Ireland, J. \& Snowdem, P. (2002). Bullying in secure hospitals. The Journal of Forense Psychiatry, 13, (3), 538-554.

Leymann, H. (1996). Contenido y desarrollo del acoso grupal/moral "Mobbing" en el trabajo. Universidad de Umea (Suecia). European Journal of Work and Organizacional Psychology, 5 (2), 165-184.

Leymann, H. (1997). The mobbing Enciclopedia. Bullying. The Definition of Mobbing at Workplace. Madrid: Pirámide.

Luna, A. (2003). Acoso psicológico en el trabajo (mobbing). Secretaría de Salud Laboral, Madrid: Ediciones GPS.

Lutgen-Sandvik, P. (2003). The communicative cycle of employee emotional abuse. Generation and regeneration of workplace mistreatment. Management Communication Quarterly, 16 (4), 471-501.

Martín-Albo, J., Núñez, J. L., Navarro, J. G., Grijalvo, F. (2007). The Rosenberg Self-Esteem Scale: Translation and Validation in University Students. The Spanish Journal of Psychology, 10 (2), 458-467.

Martínez, P. (2006). El método de estudio de caso: Estrategia metodológica de la investigación científica. Revista Pensamiento y gestión, (20). Barcelona. España.

Martínez, S. \& Cruz, M. (2008). Acoso psicológico en el trabajo. Psicología y Salud, 18 (2), 255-265.
Matthiensen, S. \& Einarsen, S. (2001). MMPI-2 configurations among victims of billying at work. European Journal of Work and Organizational Psychology, 10, 467-484.

Ministerio de la Protección Social. (2004). Violencia en el trabajo. Formas y consecuencias de la violencia en el trabajo. Medellín, Colombia: Universidad de Antioquia.

Organización Internacional del Trabajo. (2000). Las Normas Internacionales del Trabajo. México: Alfa Omega.

Pando, M., Aranda, C., Aldrete, G. \& Reynaga, E. (2006). Autoestima y redes sociales de apoyo como factores protectores de mobbing en docentes. Revista de salud pública y nutrición -Respyn, 7, (2).

Peralta, M. (2006). Manifestaciones del acoso laboral, mobbing y síntomas asociados al estrés postraumático: Estudio de caso. Revista Psicología del Caribe, 17. Universidad de la Sabana. Bogotá. Colombia.

Piñuel \& Zavala, I. (2001). Mobbing. Cómo sobrevivir al acoso psicológico en el trabajo, (pp. 58, 78, 89, 104-112, 154-166, 173, 182). Madrid: Sal Térrae.

Piñuel \& Zavala, I. (2003). Resultados del barómetro Cisneros II sobre violencia en el entorno laboral (pp. 89-95). Alcalá de Henares: Universidad de Alcalá.

Royal College of Nursing. (2005). Dealing with harassment and bullying at work: a guide: for $R C N$ members. Recuperado el 05 de octubre de 2008, disponible en: http://www.rcn.org.uk/ members/downloads/bullying-members.pdf

Sánchez, E. (1999). Relación entre la autoestima personal, la autoestima colectiva y la participación en la comunidad. Anales de Psicología, 15 (2), 251-260. Universidad de Marcia. España. 
Trujillo, M., Valderrabano, M. \& Hernández, R. (2007). Mobbing: historia, causas, efectos, y propuesta de un modelo para las organizaciones mexicanas. Revista de Ciencias Administrativas y Sociales, INNOVAR, 17, (029), 71-91.
Vargas, E. \& Barrera, F. (2005). ¿Es la autoestima una variable relevante para los programas de prevención del inicio temprano de actividad sexual? Revista, Acta Colombiana de Psicología, 13, 133-161. 